\title{
Do supine oblique views provide better imaging of the cervicothoracic junction than swimmer's views?
}

\author{
Alastair J Ireland, Ingrid Britton, Alastair W Forrester
}

\begin{abstract}
Objective-To determine whether a swimmer's view or supine (trauma) oblique views are more likely to visualise the lower cervical spine when a lateral view fails to show the cervicothoracic junction.
\end{abstract}

Design-A prospective study comparing two 20 week periods. In the first phase the swimmer's view was performed as an additional view when the cervicothoracic junction was not demonstrated. In the second phase paired supine oblique views replaced the swimmer's view.

Results-230 patients were included in the first phase, of whom 60 required swimmer's views. In the second phase 62 of 197 patients required supine oblique views. Radiology analysis of $\mathbf{5 3}$ pairs of supine oblique views showed that the vertebral bodies were adequately demonstrated at the cervicothoracic junction in only 20 patients $(38 \%)$ compared with 22 in the swimmer's group (37\%). The facet joints and posterior elements were, however, clearly seen in $37(70 \%)$ of the supine oblique patients compared with $22(37 \%)$ of the swimmer's group $\left(p<0.001, \chi^{2}\right.$ test). Exposure dose calculations showed a substantial reduction for a pair of supine oblique views (1.6 $\mathrm{mGy}$ ) over a single swimmer's view (7.2 mGy).

Conclusions-In injured patients for whom the standard three view series fails to demonstrate the cervicothoracic junction, swimmer's views and supine oblique views show the alignment of the vertebral bodies with equal frequency. However, supine oblique films are safer, expose patients to less radiation, and are more often successful in demonstrating the posterior elements.

(f Accid Emerg Med 1998;15:151-154)

Keywords: cervical spine; radiology; swimmer's view; supine oblique view

High quality radiographs of the entire cervical spine are essential in the early management of acutely traumatised patients. However, resuscitation room staff know that it is often very difficult and time consuming to obtain adequate views of the cervicothoracic junction. A widely accepted approach in the United Kingdom is distal shoulder distraction during lateral exposure followed, if this proves insufficient, by swimmer's views. Shoulder distraction may be uncomfortable and result in unwanted move- ment of the patient's neck. ${ }^{1}$ It is also possible to damage axillary vessels during shoulder movement. Similarly, the swimmer's view requires the patient to be moved during positioning, which is often difficult in multiply injured or unconscious patients, so views are often suboptimal. ${ }^{2}$

Supine oblique views, also known as "trauma obliques," taken at $30^{\circ}$ from the horizontal plane have been advocated as an alternative to the swimmer's view. ${ }^{3}$ In some centres in the United States they have been adopted as a standard view in addition to the routine three views for traumatised patients (cross table lateral, anteroposterior, and open mouth odontoid projections). ${ }^{45}$ These views have both practical and imaging advantages. Their practical advantage is based on minimal requirement for patient movement. The film cassette is placed flat on the trolley beside the patient and slid under the shoulder. With regard to the imaging advantages, additional information is obtained relating to the posterior vertebral elements (pedicles, laminae, neural foramina, and articular facets), information not provided by a further lateral view (fig 1 ).

We aimed to establish whether the supine oblique or the swimmer's view provided superior visualisation of the cervicothoracic junction where standard three view series had failed.

\section{Methods}

Ethics approval was granted by Glasgow Royal Infirmary University NHS Trust research and ethics committee. A two phase prospective study was performed, consisting of two 20 week periods. Consecutive trolley patients in the accident and emergency (A\&E) department requiring supine cervical spine radiographs for acute trauma were included. In the first phase when the standard three view series with shoulder distraction failed to demonstrate the cervicothoracic junction, swimmer's views were performed. Radiography and medical staff were already accustomed to swimmer's views, which were standard practice in the department. Before the introduction of supine oblique views, the radiographers and $A \& E$ doctors underwent a 12 week training period to ensure familiarity with the new projection. In phase two, supine oblique radiographs were performed when the standard three view series with shoulder distraction was inadequate.

Oblique views were taken with the patient supine. The cassette was placed adjacent to the side of interest with the uppermost part aligned to the top of the ear and the lowermost part slid 


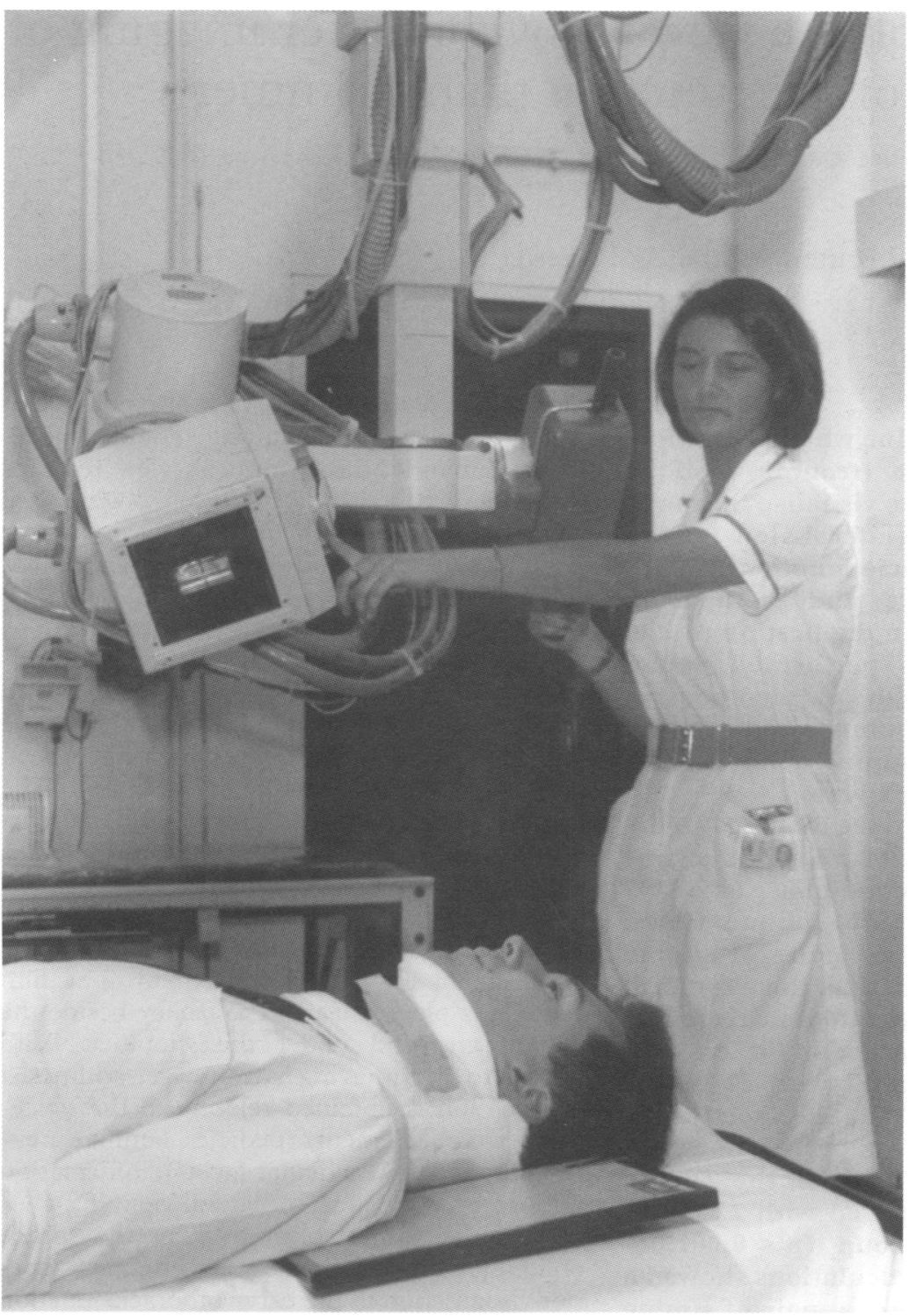

Figure 1 Radiographer taking supine oblique view. There is no need to move the patient to take supine oblique radiographs.

Table 1 Radiologist's criteria for film acceptability

\begin{tabular}{ll}
\hline AP upper cervical spine & $\begin{array}{l}\text { 1. Odontoid peg in its entirety with minimal overlapping from base of } \\
\text { skull or teeth }\end{array}$ \\
AP lower cervical spine & 1. Cateral masses of the atlanto-axial joint \\
Lateral cervical spine & 2. C3-C7 uncinate processes \\
1. Anterior arch of C1 & 2. Odontoid peg/base of skull \\
3. C1-C7 facet joints \\
4. C1-T1 spinous processes \\
5. C7-T1 joint space demonstrated at its lowest extent \\
6. Prevertebral soft tissues \\
1. Anterior arch of C1 \\
2. C2 odontoid peg \\
3. C1-T1 intervertebral foramina and their bony margins (uncinate \\
processes, superior and inferior pedicles, facet joints) \\
4. C1-T1 articular masses and laminae \\
5. C1-T1 spinous processes \\
6. C7-T1 joint space demonstrated at its lowest extent \\
1. C7-T1 vertebral bodies \\
2. C7-T1 intervertebral foramina and their bony margins (uncinate \\
processes, superior and inferior pedicles, facet joints)
\end{tabular}

under the shoulder. The beam was angled $30^{\circ}$ to the horizontal, centred on C4 (thyroid cartilage) with a film focus distance of $180 \mathrm{~cm}$ (fig 2 ). Films were subsequently analysed by a senior registrar in radiology (IB). The objective criteria used for radiological assessment of films are summarised in table 1 .

\section{Results}

Four hundred and twenty seven patients requiring cervical spine radiology for acute trauma were included in the study. Sixty of 230 patients in phase one (26\%) required swimmer's views, compared with 62 of 197 patients in phase two (32\%) who required supine oblique views (NS).

Following radiology analysis, the vertebral bodies and posterior elements of the cervicothoracic junction were judged to have been satisfactorily imaged in $37 \%$ of the swimmer's group (22/60 patients) and $38 \%$ of the supine oblique group (20/53 patients) (NS). However, where the swimmer's view failed to demonstrate the cervicothoracic junction, it also failed to provide any information about the posterior elements. In contrast, where supine oblique views failed to show the vertebral bodies satisfactorily, useful information could still be obtained about the posterior elements $(70 \% v$ $37 \%, \mathrm{p}<0.001, \chi^{2}$ test) (table 2 ).

No statistically significant difference was found between the two groups in the number of patients requiring a repeat additional view. $A \& E$ radiographers rejected the initial exposure of swimmer's views in 34 patients (56\%), compared with 26 patients $(41 \%)$ whose initial supine oblique films were radiographically rejected $\left(p=0.1, \chi^{2}\right.$ test). However, the overall number of exposures required to obtain a satisfactory cervical spine series was found to be significantly lower in the supine oblique group (acceptance rate $127 / 174(71 \%)$ for supine oblique views $v 61 / 106$ (58\%) for swimmer's view; $\mathrm{p}<0.05, \chi^{2}$ test with Yates correction).

The standard exposure doses were calculated for the different views. Despite replacing a single view with a pair of views, there was a substantial reduction in the radiation exposure for a pair of supine oblique films (1.6 mGy) over a single swimmer's view $(7.2 \mathrm{mGy})$. Therefore in addition to reducing the total number of exposures, for any one patient a radiation dose reduction was achieved for the cervical spine series in the supine oblique group.

\section{Discussion}

We found that the vertebral body alignment was demonstrated with equal frequency in both views. However, supine oblique views visualise the posterior elements of the cervicothoracic junction more reliably than the swimmer's view.

Table 2 Patients'films judged technically adequate by radiologist

\begin{tabular}{lll}
\hline & $\begin{array}{l}\text { Vertebral bodies and } \\
\text { posterior elements }\end{array}$ & $\begin{array}{l}\text { Posterior } \\
\text { elements }\end{array}$ \\
\hline Swimmer's & $37 \%(22 / 60)$ & $37 \%(22 / 60)$ \\
Supine oblique & $38 \%(20 / 53)$ & $70 \%(37 / 53)$ \\
\hline
\end{tabular}



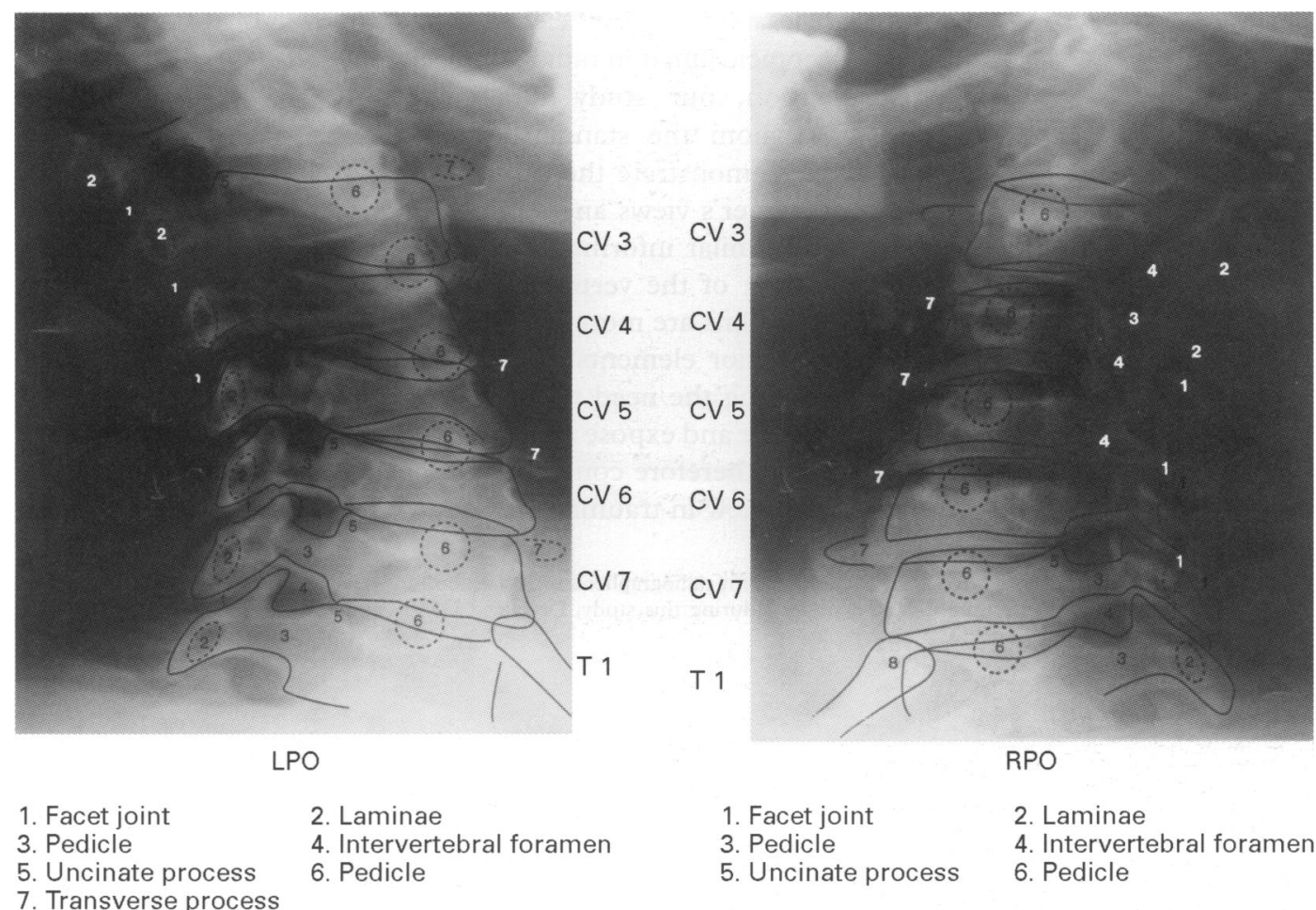
1. Facet joint
3. Pedicle
5. Uncinate process
2. Laminae
4. Intervertebral foramen
6. Pedicle

7. Transverse process

Figure 2 Paired supine oblique views. Interpretation of supine oblique views should concentrate on posterior elements. "Roof tiling" of the facet joints and laminae should be maintained throughout. The pedicles should maintain parallel alignment with progressive gradation of the intervertebral foramina. LPO, left posterior oblique; RPO, right posterior oblique.

Supine oblique views also have the advantage of improved safety and reduced radiation dose.

There may be several potential sources of bias within the study. The radiographers were aware of the study objectives with regard to the visualisation of the cervicothoracic junction, and this may have influenced the overall rate of visualisation. It should not, however, have biased either limb of the study, as they were unaware that the new view was to be compared with the swimmer's view. The 12 week training period between the two phases should have eliminated any increased reject rate resulting from a learning curve.

The radiographically accepted films were initially reported for the purposes of clinical management by the general radiology department. They were subsequently recalled and along with all the radiographically rejected films, assessed for technical acceptability according to the objective radiological criteria already outlined. Potential bias arises in a single radiologist (IB), who was aware of the study objective, analysing these films, although this was minimised by the use of unambiguous criteria.

Nine patients' supine oblique films were unavailable for technical analysis. All nine patients' films were untraceable during follow up for non-cervical injuries. None had a significant radiographic abnormality detected on presentation or had further investigation of cervical symptoms. A selection bias in terms of radiographic difficulty was not substantiated on review of the number of exposures required. (The mean number of exposures for the missing nine patients was 6.4 , while the mean number of exposures for the available supine oblique series was 8.0.)
Various studies have shown the inadequacy of current approaches to imaging the cervical spine. Moulton and Griffiths ${ }^{6}$ showed that only $75 \%$ of radiographs adequately demonstrated the cervical spine even after further radiographic examinations (not including supine obliques). If adequate, the lateral radiograph alone will detect $70-80 \%$ of cervical spine injuries, while the standard three views were found to be $93 \%$ sensitive in comparison with thin section tomography. ${ }^{7}$ Turetsky et al, using supine oblique radiographs at $30^{\circ}$, demonstrated a further $7 \%$ with cervical spine injury in whom the standard three views showed no injury. ${ }^{4}$

Our study confirms that where the standard three view series fails to demonstrate the cervicothoracic junction, the swimmer's view retains a role in assessing vertebral body alignment and integrity. However, supine obliques are performed without moving the patient and expose patients to less radiation, and so they are safer. The avoidance of unnecessary movement is an important advantage, particularly in severely traumatised individuals who may either experience pain during shoulder distraction or suffer further occult injury. Because acceptable films are easier to obtain, they may be superior to swimmer's views in the unconscious or severely injured patient in whom radiography and positioning are difficult.

Supine obliques are significantly more likely to demonstrate the posterior cervical elements and their alignment at the cervicothoracic junction. Harris and Mirvis suggest that when integrated with an adequate anteroposterior projection of the lower cervical spine, right and left supine oblique projections provide sufficient information about the posterior vertebral 
bodies, pedicles, and posterior elements to clear the cervicothoracic junction radiographically. ${ }^{8}$

In conclusion, our study shows that in patients for whom the standard three view series fails to demonstrate the cervicothoracic junction, swimmer's views and supine oblique views provide similar information with regard to the alignment of the vertebral bodies, but supine oblique films are more likely to demonstrate the posterior elements and their alignment. By avoiding the need to move patients, they are also safer and expose the patient to less radiation. They therefore constitute an invaluable additional view in trauma radiology.

We thank superintendent radiographer Lorna Turnbull for her invaluable assistance during this study, Dr David Gentle and
Mr Ian Kennedy of the West of Scotland Health Boards Department of Clinical Physics and Bio-engineering for data on radiation exposure doses, and Miss Jackie Riley of Glasgow Caledonian University for statistical advice.

1 Kaufman $\mathrm{HH}$, Harris JH, Spencer JA, Kopansisky DR. Danger of traction during radiography for cervical trauma. JAMA 1982;247:2369.

2 Harris JH, Mirvis SE. The radiology of acute cervical spine trauma, 3rd ed. Baltimore: Williams and Wilkins, 1996 195.

3 Daffner RH. Evaluation of cervical vertebral injuries. Semin Roentgenol 1992;27:239-53.

4 Turetsky DB, Vines FS, Clayman DA, Northup HM. Technique and use of supine oblique views in acute cervical nique and use of supine oblique views in acu
spine trauma. Ann Emerg Med 1993;22:685-9.

5 Murphey MD. Trauma oblique cervical spine radiographs. Murphey MD. Trauma oblique cer
Ann Emerg Med 1993;22:728-30.

6 Moulton C, Griffiths PD. The adequacy of cervical spine radiographs in the accident and emergency department. J R Soc Med 1993;86:141-3.

7 Streitweiser DR, Knopp R, Wales LR, Williams JL, Tonnemacher K. Accuracy of standard radiographic views of detecting cervical fractures. Ann Emerg Med 1983;12: 538-42.

8 Harris JH, Mirvis SE. The radiology of acute cervical spine trauma, 3rd ed. Baltimore: Wlliams and Wilkins, 1996:206.

\section{First Asian Conference on Emergency Medicine, 22-25 October 1998}

The Society for Emergency Medicine in Singapore is privileged to host the First Asian Conference on Emergency Medicine. The conference is scheduled to be held on 22-25 October 1998. This conference will also see the official launch of the Asian Society of Emergency Medicine.

The Committee will be inviting world renowned experts to deliver lectures on prehospital trauma care, advanced imaging for multiply injured, aeromedical evacuation of the trauma patient, development of emergency medicine, issues and challenges for emergency medicine, emergency nurse triage, advanced training for emergency nursing, development of clinical practice guidelines, emergency department design, management issues at the emergency department, update on cardiac resuscitation, current trends on GI decontamination, elements of EMS, and prehospital triage and IT in emergency medicine. The preconference workshops will be held in the same venue, on focused ultrasound for the emergency physician, advanced airway management, and local anaesthesia techniques.

For further information, contact the Conference Secretariat, Academy of Medicine, Singapore, 16 College Road, \#01-01 College of Medicine Building, Singapore 169854; tel +65 223 8968; fax +65 225 5155; email: monicaw@pacific.net.sg 